\title{
Grinding Chatter Detection and Identification Based on BEMD and LSSVM
}

\author{
Huan-Guo Chen ${ }^{1}$, Jian-Yang Shen ${ }^{2}$, Wen-Hua Chen ${ }^{1 *}$, Chun-Shao Huang ${ }^{3}$, Yong-Yu Yi $^{1}$ and Jia-Cheng Qian ${ }^{1}$
}

\begin{abstract}
Grinding chatter is a self-induced vibration which is unfavorable to precision machining processes. This paper proposes a forecasting method for grinding state identification based on bivarition empirical mode decomposition (BEMD) and least squares support vector machine (LSSVM), which allows the monitoring of grinding chatter over time. BEMD is a promising technique in signal processing research which involves the decomposition of two-dimensional signals into a series of bivarition intrinsic mode functions (BIMFs). BEMD and the extraction criterion of its true BIMFs are investigated by processing a complex-value simulation chatter signal. Then the feature vectors which are employed as an amplification for the chatter premonition are discussed. Furthermore, the methodology is tested and validated by experimental data collected from a CNC guideway grinder KD4020X16 in Hangzhou Hangji Machine Tool Co., Ltd. The results illustrate that the BEMD is a superior method in terms of processing non-stationary and nonlinear signals. Meanwhile, the peak to peak, real-time standard deviation and instantaneous energy are proven to be effective feature vectors which reflect the different grinding states. Finally, a LSSVM model is established for grinding status classification based on feature vectors, giving a prediction accuracy rate of 96\%.
\end{abstract}

Keywords: Grinding chatter, BEMD and LSSVM, Complex-value chatter signal, Feature vector, Grinding status classification

\section{Introduction}

Grinding is an abrasive machining process which is widely used in modern manufacturing practice to produce high surface quality and close tolerance [1-4]. Particularly with the increasing mature of ultra-high speed grinding, its advantages are further improved, that providing convenient conditions for development of aerospace technology, transportation, military and other industries $[5,6]$. However, grinding chatter is one of the most unfavorable dynamic phenomena in grinding operations including regenerative chatter, frictional chatter and mode coupling chatter. In practice, grinding chatter has negative impacts on the ultimate geometrical workpiece accuracy, surface quality and productivity of machinery. Moreover, it leads to increased wheel wear and adds time and costs to manufacturing [7, 8]. Many

\footnotetext{
*Correspondence: chenwh@zstu.edu.cn

1 Zhejiang Province's Key Laboratory of Reliability Technology for Mechanical and Electrical Product, Hangzhou 310018, China Full list of author information is available at the end of the article
}

theories have been proposed and experiments carried out to discover exactly what mechanism underlies grinding chatter, with the aim of developing reliable suppression methods subsequently [9].

At present, only a few methods for chatter detection have been successfully and practically applied in industry. It is common for trained machine operators to identify the appearance of chatter through experience or observation, meaning that corresponding measurements are not taken at the time that resulting in irreparable loss for the industry. Signal processing techniques and appropriate feature vectors are very important for chatter detection. In the past few decades, either nonlinear time series modeling [10] or spectral analysis [11, 12] has been applied for chatter detection. Additionally, Tansel et al. [13], adopted s-transformation to extract the damping index, making a very descriptive feature of chatter available for inspection in turning operations. Yao et al. [14], presented a two-dimensional feature vector for chatter detection based on the standard deviation of wavelet transforms in drilling machining which had 
an advantageous identification time. In another study, Gradisek et al. [15] used the coarse-grained entropy rate as a chatter index in grinding and turning, as its value exhibits a drastic drop at the onset of chatter.

It is important to note that the signal processing methods proposed above were mostly based on the theory of Fourier transformation and that these traditional methods are not applicable to processing grinding signals (which are almost non-stationary and nonlinear). They can only detect chatter if it is already in an almost fully developed stage and easily to extract spurious frequency and error information from chatter signals. In order to highly meet the demand of real-world production, it is necessary to detect the onset of chatter before chatter marks have been made on the workpiece. Given this requirement, Rilling et al. [16] proposed a novel method called the bivarition empirical mode decomposition (BEMD). In the third session of the HHT (HilbertHuang Transform) International conference, BEMD was successfully applied to the monitoring of wind turbine conditions and displayed its feasibility as a method to determine weak features and integrate information from non-stationary and nonlinear signals [17].

The author of this paper also has made a comparison between EMD and BEMD in extracting features for grinding chatter signals to show the advanced performance of BEMD, that the paper is accepted by the 2016 11th International Conference on Reliability, Maintainability and Safety (ICRMS' 2016). Thus will not be repeated in details here and just give out some brief conclusions about the distinctions between EMD and BEMD: (1) EMD is initially applied to a one-dimensional signal and extracts zero-mean oscillating components, whereas BEMD is applied to a bivariate signal and extracts zero-mean rotating components; (2) BEMD has calculation efficiency due to process complex-value signals simultaneously and only compute the upper envelope using the maximum points, while EMD can only decompose signals one-by-one and has to obtain both upper and lower envelopes by connecting the extreme points; (3) The number of IMFs derived from signals by EMD are different, and can't reveal any synchronous characteristics and phase shifting, nor can EMD extract an information fusion function. While the number of IMFs by BEMD is the same, it can extract an information fusion function well and preserve phase differences; (4) BEMD has facilitates the establishment of purified shaft vibration orbits and fully guarantees the correctness of results, which EMD cannot.

Additionally, there are several smart classifiers essential for grinding state identification, such as artificial neural network (ANN) [18, 19], fuzzy logic and support vector machines (SVM). Li et al. [20] used multilayer perceptron
ANN to distinguish the tool breakage and cutting chatter. According to the trend of signal in time domain. Bediaga, et al. [21] established the fuzzy logical rule to analyze stability of cutting system. Moreover, Jiang et al. [22] adopted multi-class SVM to identify and classify cutting states that accuracy rate reached $95 \%$. The ANN usually suffers from the problem of intrinsic defeats such as slow study speed, multiple local minima and over-fitting. Also, the prediction ability of fuzzy logic is inaccurate and its theory is still imperfect. SVM overcomes these deficiencies by using the structural risk minimization principle to enhance extensive ability and it also stresses the study of statistical learning rules with a small sample. In order to further improve the learning speed [23]. Suykens proposed a modified version of SVM, i.e. the least squares SVM (LSSVM). In the LSSVM, the non-sensitive loss function is replaced by a quadratic loss function and the inequality constraints are replaced by equality constraints. Through constructing a loss function, the quadratic programming problem is translated into solving linear equation group problems, which simplifies the complexity of calculation $[24,25]$.

The advantages of BEMD and LSSVM are combined in this paper for detecting and identifying grinding chatter. Section two gives a brief review of BEMD and LSSVM, as well as the extraction criterion of true BIMFs. Moreover, the peak to peak, real-time standard deviation and instantaneous energy are presented as feature vectors for the grinding chatter. In section three, a simulation chatter signal is constructed and then processed by BEMD. Afterwards, peak to peak, real-time standard deviation and instantaneous energy are extracted from BIMFs. In section four, the benefits of the proposed method are further validated experimentally by processing grinding signals which are derived from the grinder KD4020X16, and then a LSSVM model is established to predict the grinding state. Finally, conclusions are presented in section five, which also gives new directions for future work.

\section{BEMD and LSSVM}

\subsection{A Brief Review of BEMD}

\subsubsection{Algorithm of BEMD}

The BEMD is proposed as an enhancement of EMD while inheriting all of its merits [26, 27]. BEMD is devoted to decomposing sequentially a complex-value signal into a collection of complex-value BIMFs which range from high frequency to low frequency, plus a non-zero mean low-degree polynomial remainder named the residue. Generally, the residue is considered as the trend of the signal. All BIMFs derived from the grinding signals are characterized as rotation components. Each of the BIMFs must satisfy the following conditions. 
(1) The number of extrema and zeros must be equal or different at most by one.

(2) The mean value of the envelope at any point defined by the local maximum points and the envelope as defined by the local minima must be zero.

The fundamental sifting process of BEMD can be depicted as follows.

$\mathrm{S}_{1} \quad$ Select a bivariate signal $s(t)=x(t)+i y(t)$ and a set of projection directions: $\varphi_{k}=2 k \pi / N, 1 \leq k \leq N$

$\mathrm{S}_{2} \quad$ For $1 \leq k \leq N$.

$\mathrm{S}_{21}$ Project the signal $s(t)$ on directions $\varphi_{k}$ :

$p_{\varphi_{k}}=\operatorname{Re}\left[s(t) \exp \left(-i \varphi_{k}\right)\right]$

$\mathrm{S}_{22}$ Extract all partial maximum points of $p_{\varphi_{k}}(t)$ : $\left\{\left(t_{i}^{k}, p_{i}^{k}\right)\right\}$, where $i$ indicates number of individual maxima.

$\mathrm{S}_{23}$ Interpolate the set of points $\left\{\left(t_{i}^{k}, p_{i}^{k} \exp \left(i \varphi_{k}\right)\right)\right\}$ by cubic spline interpolation to obtain the partial envelope curve in direction $\varphi_{k}$, namely, $e_{\varphi_{k}}(t)$.

$\mathrm{S}_{3}$ Calculate the mean of all envelop curves:

$\bar{m}(t)=\frac{1}{N} \sum_{k=1}^{N} e_{\varphi_{k}}(t)$,

$\mathrm{S}_{4} \quad$ Subtract the mean $\bar{m}(t)$ from $s(t)$ to obtain $g(t)$ :

$g(t)=s(t)-\bar{m}(t)$.

$\mathrm{S}_{5}$ Examine if $g(t)$ is a BIMF:

$\mathrm{S}_{51}$ If not, replace $s(t)$ by $g(t)$ and repeat the procedure from step $\mathrm{S}_{2}$ until $g(t)$ is a BIMF.

$\mathrm{S}_{52}$ If it is, record the obtained BIMF and repeat the procedure from step $\mathrm{S}_{2}$ on the residual signal $g(t)$.

As well as referring to the sifting process of BEMD, the $s(t)$ can be expressed by the procedure:

$$
s(t)=\sum_{m=1}^{n} g_{m}(t)+r_{n}(t),
$$

where $g_{m}(t)$ denotes the $m$ th complex-valued BIMF and $r_{n}(t)$ denotes the residue.

\subsubsection{Extraction Criteria of True BIMFs}

It is worth noting that the above-generated BIMFs basically incorporate two components: true BIMFs and spurious BIMFs. These spurious BIMFs cannot exactly reflect the vibration peculiarities of grinding systems in a physical sense and this seriously interferes with the researchers' efforts to extract the feature vectors from signals and eliminate the mechanism faults of grinders. In general, the generation of spurious BIMFs are summarized by the following factors: (1) the definition of BIMFs is only based on numerical analysis, without referring to its physical significance; (2) the stopping criterion of the sifting process results in an excessive decomposition phenomenon; (3) end effect which can lead to serious deviation from the actual features of the signal is not fully eliminated; (4) either white noise or pulse interference which is superimposed on the vibration signal may produce high frequency spurious components. Considering that the majority of people rely heavily on their experience to estimate the authenticity of BIMFs, this is not conducive to facilitating the expansion of the BEMD method. It is therefore necessary to use an efficient and reliable method to identify and eliminate the spurious BIMFs, a procedure which is of great importance to the extraction of the actual vibration mode and corresponding features of the time-frequency domain.

As the BIMFs are recognized as the orthogonal expression of the signal, the true BIMFs will have a higher correlation with the original signal compared to the spurious BIMFs. Therefore, it is reasonable to employ the correlation coefficient as a measurement index to remove the spurious components and then classify it as part of a residual [28]. The specific sifting process could be described as in Table 1 , if the correlation coefficient of each complex-value BIMF $\zeta_{m}=\sqrt{\left|\zeta_{m}^{1}\right| \times\left|\zeta_{m}^{2}\right|},(m=1,2, \ldots, n)$, has been obtained (where, $\zeta_{m}^{1}$ and $\zeta_{m}^{2}$ denote the correlation coefficient of the real and imaginary parts of BIMFs, respectively).

The $\eta$ in Table 1 is a fixed threshold that is generally adopted as a ratio of the maximum correlation coefficient, where $\delta$ is a ratio coefficient larger than 1 :

$$
\eta=\max \left(\zeta_{m} / \delta\right), \quad m=1,2, \ldots, n .
$$

\subsection{Brief Review of LSSVM}

The LSSVM is an improved algorithm based upon standard SVM that a two-norm is taken with equality instead of inequality constraints so as to obtain a linear set of equations instead of a quadratic programming problem in the dual space $[29,30]$. It shows specific powerful capability in processing small sample, nonlinear and high-dimension pattern. The formulation of LSSVM can be described as follows:

\section{Table 1 Extraction criterion of true BIMFs based on correlation coefficient}

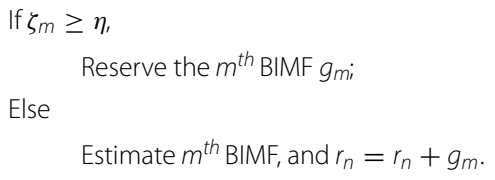


(1) Using a training set of the data points $D=\left\{\left(x_{i}, y_{i}\right) \mid i=1,2, \ldots, n\right\}$, where $x_{i} \in R^{n}$ is the $i$ th input data, and $y_{i} \in\{-1,+1\}$ is the output class.

(2) The regression function in high-dimensional space is constructed:

$y(x)=\boldsymbol{\omega} \cdot \varphi(x)+b$,

where $\boldsymbol{\omega}$ is the weight vector, $\varphi(x)$ is a nonlinear function that maps the input data $x$ into a lowdimension space and $b$ is the bias parameter.

(3) According to the structural risk minimization principle, the optimal $\omega$ and $b$ can be obtained by minimizing the following function:

$$
\begin{aligned}
\min _{\omega, b, \varepsilon} J(\boldsymbol{\omega}, \varepsilon) & =\frac{1}{2}\|\boldsymbol{\omega}\|^{2}+\frac{C}{2} \sum_{i=1}^{n} \varepsilon_{i}^{2}, \\
\text { s.t. } y_{i} & =\boldsymbol{\omega} \cdot \varphi\left(x_{i}\right)+b+\varepsilon_{i},
\end{aligned}
$$

where $C$ is the penalty coefficient to balance the structural risk and experience risk and $\varepsilon_{i}$ is the slack variable.

(4) The Lagrange function can be constructed to solve the optimization problem:

$L(\boldsymbol{\omega}, b, \varepsilon, \alpha)=J(\boldsymbol{\omega}, \varepsilon)-\sum_{i=1}^{n} \alpha_{i}\left(\boldsymbol{\omega} \cdot \varphi\left(x_{i}\right)+b+\varepsilon_{i}-y_{i}\right)$,

where $\alpha_{i}$ represent Lagrange multipliers that can be either positive or negative values. Eq. (8) can be changed to the following equivalent equations:

$$
\left\{\begin{array}{l}
\frac{\partial L}{\partial \omega}=0 \Rightarrow \omega-\sum_{i=1}^{n} \alpha_{i} \varphi\left(x_{i}\right)=0, \\
\frac{\partial L}{\partial b}=0 \Rightarrow \sum_{i=1}^{n} \alpha_{i}=0, \\
\frac{\partial L}{\partial \varepsilon_{i}}=0 \Rightarrow C \varepsilon_{i}-\alpha_{i}=0, \\
\frac{\partial L}{\partial \alpha_{i}}=0 \Rightarrow \omega \cdot \varphi\left(x_{i}\right)+b+\varepsilon_{i}-y_{i}=0 .
\end{array}\right.
$$

(5) Eliminating $\boldsymbol{\omega}$ and $\varepsilon_{i}$ and expressing in matrix form gives:

$$
\left[\begin{array}{lc}
0 & \boldsymbol{e}^{\mathrm{T}} \\
\boldsymbol{e} & \boldsymbol{\Omega}_{i, j}+\boldsymbol{C}^{-1} \boldsymbol{I}
\end{array}\right]\left[\begin{array}{l}
b \\
\boldsymbol{\alpha}
\end{array}\right]=\left[\begin{array}{l}
0 \\
\boldsymbol{y}
\end{array}\right]
$$

where

$$
\begin{aligned}
& \boldsymbol{e}=[1,1, \ldots, 1]_{n}^{\mathrm{T}}, \\
& \boldsymbol{y}=\left[y_{1}, y_{2}, \ldots, y_{n}\right], \\
& \boldsymbol{\alpha}=\left[\alpha_{1}, \alpha_{2}, \ldots, \alpha_{n}\right]^{\mathrm{T}},
\end{aligned}
$$

$\boldsymbol{\Omega}_{i, j}=\left(x_{i}\right) \times\left(x_{j}\right)=K\left(x_{i}, x_{j}\right)$ is the kernel function.

The commonly used kernel functions are listed as follows [31, 32].

Polynomial kernel function:

$$
K\left(x_{i}, x_{j}\right)=\left(\left(x_{i}, x_{j}\right)+\theta\right)^{d}, \quad d=1,2, \ldots
$$

RBF kernel function:

$$
K\left(x_{i}, x_{j}\right)=\exp \left(\frac{-\left\|x_{i}-x_{j}\right\|^{2}}{\sigma^{2}}\right) .
$$

Sigmoid kernel function:

$$
K\left(x_{i}, x_{j}\right)=\tanh \left(v\left(x_{i}, x_{j}\right)+c\right) \text {. }
$$

(6) Lastly, the linear model for function estimation is achieved after the optimization problem is solved:

$$
y(x)=\sum_{i=1}^{n} \alpha_{i} K\left(x_{i}, x_{j}\right)+b .
$$

\subsection{Chatter Feature Vectors Extraction}

Numerous experiments have shown that the amplitude of the vibration signal fluctuates within a certain range when the grinder is in a stable grinding state, while the amplitude substantially increases when in a transition state. It later becomes steady again when the grinder is in a chatter state; Therefore, early grinding chatter can be preliminary detected by comparing changes in the timedomain statistical parameters of the signal. In this paper, the peak to peak $(p p)$, real-time standard deviation $(R s d)$ and instantaneous energy (IE) are conceived as ideal feature vectors that can detect and identify the chatter.

The peak to peak represents the difference between the maximum and minimum values of the signal, which is recognized as the most intuitive indicator for amplitude change in the signal [33]:

$$
p p=\left|\left\{x_{i}\right\}\right|_{\max }-\left|\left\{x_{i}\right\}\right|_{\min }, \quad i=1,2, \ldots, N,
$$

where $N$ represents the sampling points.

The real-time standard deviation indicates the deviation degree from the mean chatter signal, which in a sense reflects the oscillation trend [34]. Rsd can be described as:

$$
\begin{aligned}
R s d^{2} & =\frac{1}{n} \sum_{i=1}^{n}\left(\left|x_{i}\right|-\bar{x}\right)^{2} \\
& =\frac{1}{n} \sum_{i=1}^{n} x_{i}^{2}-\frac{1}{n} \sum_{i=1}^{n} x_{i} \\
& =\left(\frac{x_{1}^{2}}{n}-\frac{x_{1}}{n}\right)+\left(\frac{x_{2}^{2}}{n}-\frac{x_{2}}{n}\right)+\cdots+\left(\frac{x_{n}^{2}}{n}-\frac{x_{n}}{n}\right),
\end{aligned}
$$


where $\bar{x}$ represents the mean of the signal.

In practice, any internal fluctuation may result in a vibration of instantaneous energy, which means that the change in instantaneous energy has a direct relation to abnormal system operation [35]. Thus, instantaneous energy can be defined as follows:

$$
I E=\frac{1}{2}\left|\alpha_{i}^{2}+j \psi_{i}^{2}\right|, \quad j=\sqrt{-1}, i=1,2, \ldots, N,
$$

where $\alpha_{i}$ and $\psi_{i}$ represent the instantaneous amplitude of the real and imaginary parts of signal respectively [36].

Using these definitions, the $p p$, Rsd and $I E$ of each BIMF can be monitored every second, achieving initially detecting grinding chatter in real time.

\section{Application of BEMD to Simulate Chatter Signal}

\subsection{Construction of a Simulation Chatter Signal}

In order to evaluate whether BEMD could be a reliable technique employed for grinding chatter detection, a simulation of a complex-value chatter signal $s(t)$ was constructed according to the mechanism of chatter and characteristics of the time-frequency domain:

$$
s(t)=\left\{\begin{array}{c}
x(t)+c_{1}(t)+i\left(y(t)+c_{2}(t)\right), \quad 0 \leq t \leq 2.5 \\
\left(x(t)+c_{1}(t)\right)(1+4(t-2.5))+i((y(t)+\cdots \\
\left.\cdots+c_{2}(t)\right)(1+5(t-2.5)), \quad 2.5<t \leq 3 \\
2.5\left(x(t)+c_{1}(t)\right)+i 3\left(y(t)+c_{2}(t)\right), \quad 3<t \leq 5
\end{array}\right.
$$

where

$$
\begin{aligned}
x(t) & =3 \sin (50 t)+4 \sin (100 t)+0.4, \\
y(t) & =2 \sin (50 t+4)+3 \sin (100 t+2.4)-0.2, \\
c_{1}(t) & =1.5 \operatorname{rand}(2001,1)-0.75, \\
c_{2}(t) & =\operatorname{rand}(2001,1)-0.5 .
\end{aligned}
$$

It is clearly seen that the real and imaginary parts of signal $s(t)$ are composed of two sine signals and white noise, respectively, and that the frequency components of both are $50 \mathrm{rad} / \mathrm{s}$ and $100 \mathrm{rad} / \mathrm{s}$. Additionally, the phase of the imaginary parts of signal is shifted by $0.08 \mathrm{rad}$ and 0.024 rad. The output is a harmonic vibration signal which simulates the stable grinding process when $t \leq 2.5 \mathrm{~s}$. The output is the harmonic vibration signal multiplied with a slant sign also as to simulate the grinding chatter when $2.5<t \leq 3 \mathrm{~s}$. Moreover, the output is the original harmonic vibration signal multiplied by gain coefficients in order to simulate the stable chatter status when $3<t \leq 5 \mathrm{~s}$.

The chatter signal is shown in Figure 1. The blue solid lines represent the real part of signal while the red dash lines indicate the imaginary part. It is clearly seen that the amplitude of the signal is small in a stable grinding process, while the amplitude significantly increases after $2.5 \mathrm{~s}$, then after $3 \mathrm{~s}$ the amplitude become steady as the grinder settles into stable chatter. Hence it's change trend and distribution are similar to experimental chatter images in Refs. [14, 37], that this chatter signal well simulates the chatter process.

\subsection{Application of BEMD}

Decomposing this chatter signal using BEMD sets 64 projection directions and 10 iterations, generating the BIMFs shown in Figure 2. The blue solid lines represent the real part of the BIMFs while the red dash lines indicate the imaginary part.

From Figure 2, it is seen that the simulation complexvalue chatter signal is decomposed into 6 BIMFs and a residue, which can significantly facilitate the establishment of a pure vibration orbit and fully guarantees the correctness of the established result. The extraction criterion of true BIMF introduced in Section 2.1.2 is then applied to the decomposed BIMFs and the correlation coefficients of the BIMFs are shown in Table 2.

Compared with the data in Table 2, only the first two BIMFs, which have a relatively high correlation with original signals, should be reserved. The other four BIMFs have to be removed and classified as a part of the residual. The true BIMFs which are derived from simulation chatter signals are shown in Figure 3, where the real parts of BIMFs are plotted as blue solid lines and the imaginary parts are plotted as red-dashed lines.

In Figure 3, it is clearly seen that there is mutual dependence between the real and imaginary part of the true BIMFs (i.e., BIMF 1 and $\mathrm{BIMF}_{2}$ ) and that the portions where components are rotating can be identified by a constant phase shift. The cross-correlation function (CCF) of each true BIMF could therefore be obtained and then the phase parameters could be estimated from the CCF [38], as shown in Figure 4. Moreover, the amplitude and the frequency components of the chatter signal which are initially set in the previous signal also could be revealed by carrying out the Hilbert transformation on the true BIMFs, as shown in Figure 5, where the marginal

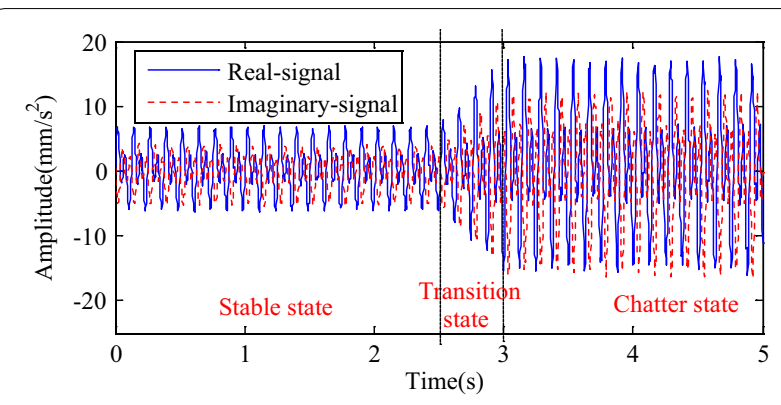

Figure 1 Complex-valued chatter signal 


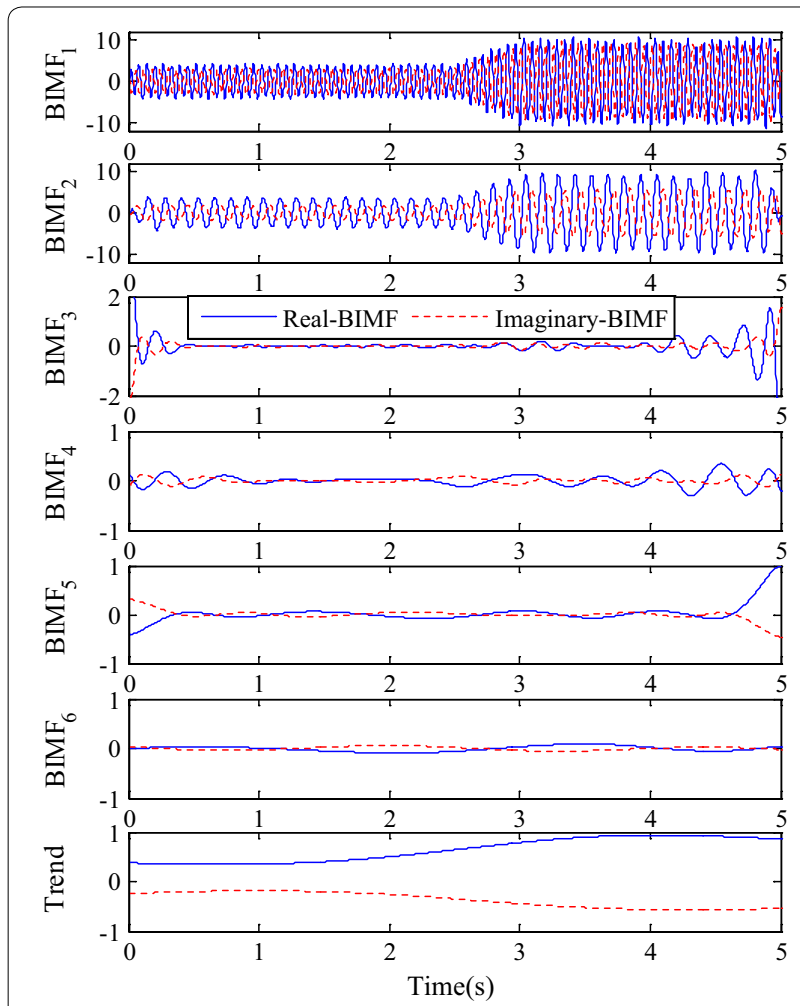

Figure 2 The so-generated BIMFs of simulation chatter signal

Table 2 Correlation coefficient of BIMFs of simulation chatter signal

\begin{tabular}{lll}
\hline No. & BIMFs & $\begin{array}{l}\text { Correlation } \\
\text { coefficient }\end{array}$ \\
\hline 1 & $\mathrm{BIMF}_{1}$ & 0.8025 \\
2 & $\mathrm{BIMF}_{2}$ & 0.6281 \\
3 & $\mathrm{BIMF}_{3}$ & 0.0526 \\
4 & $\mathrm{BIMF}_{4}$ & 0.0176 \\
5 & $\mathrm{BIMF}_{5}$ & 0.0043 \\
6 & $\mathrm{BIMF}_{6}$ & 0.0147 \\
\hline
\end{tabular}

spectrum of real part of signal is plotted as blue solid line and imaginary part of signal is plotted as red-dashed line. The marginal spectrum expresses the amplitude of each frequency in space and represents accumulated amplitude in a statistical sense.

According to Figure 4, it is clearly seen that the phase shifting and synchronization information about the real and imaginary parts of the true BIMFs are well preserved and easily detected. The phase shifting of BIMF $_{1}$ and $\mathrm{BIMF}_{2}$ is $0.025 \mathrm{rad}$ and $0.08 \mathrm{rad}$, respectively, which is similar to the phase as described in the simulation signal. From Figure 5, the frequency components of the real and imaginary parts of the signal $(8 \mathrm{~Hz}$ and $16 \mathrm{~Hz})$ are

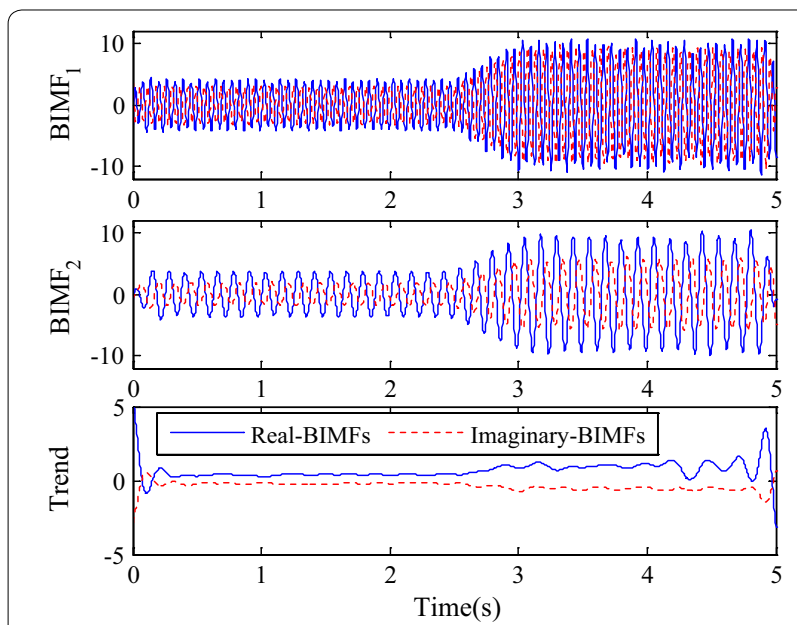

Figure 3 True BIMFs of a simulated chatter signal
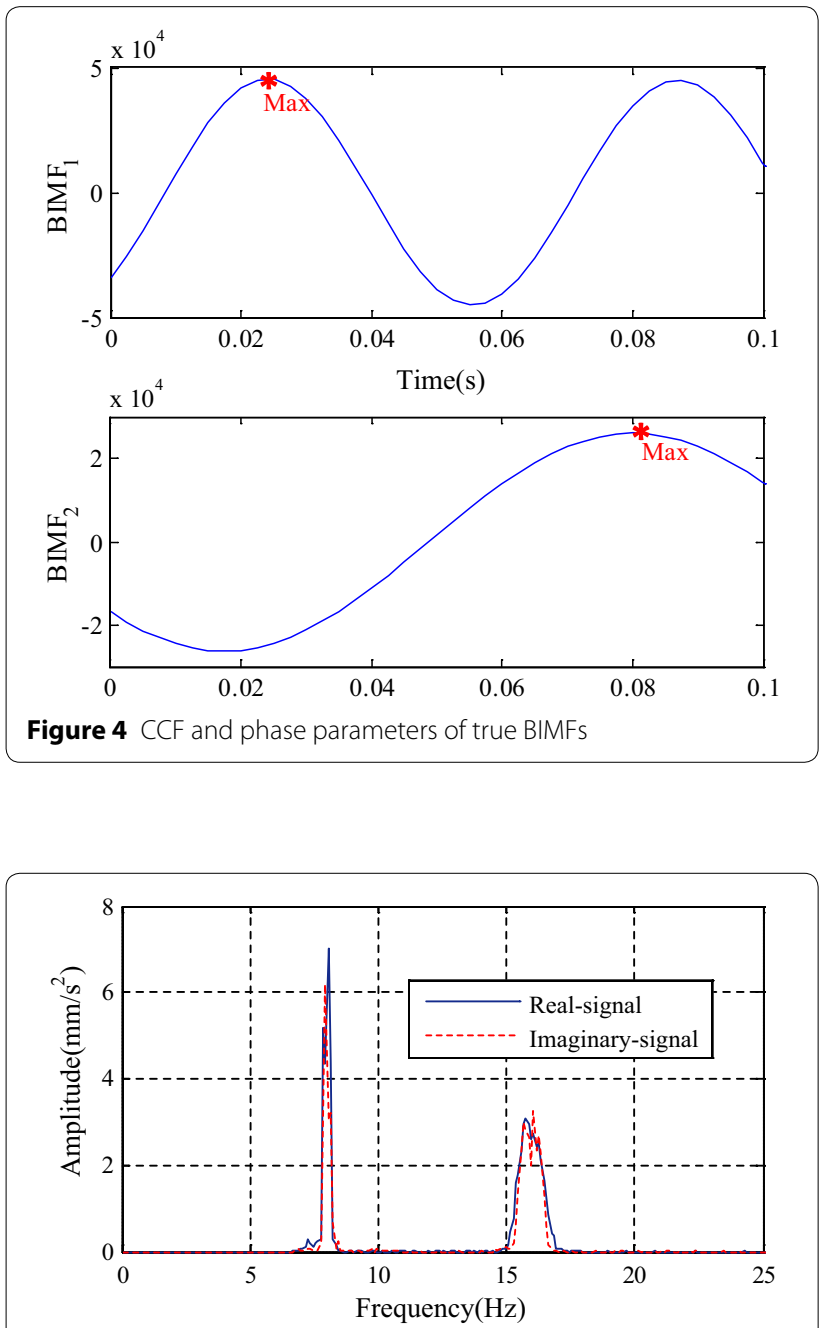

Figure 5 Marginal spectrum of the real part of signal and imaginary part of signal 


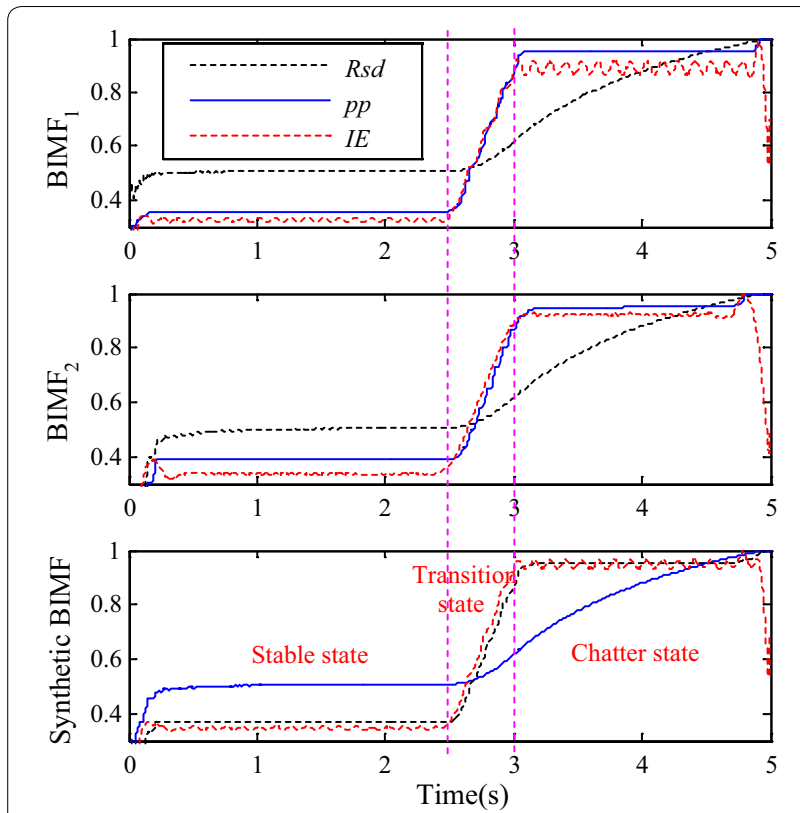

Figure 6 Feature vectors of the BIMFs

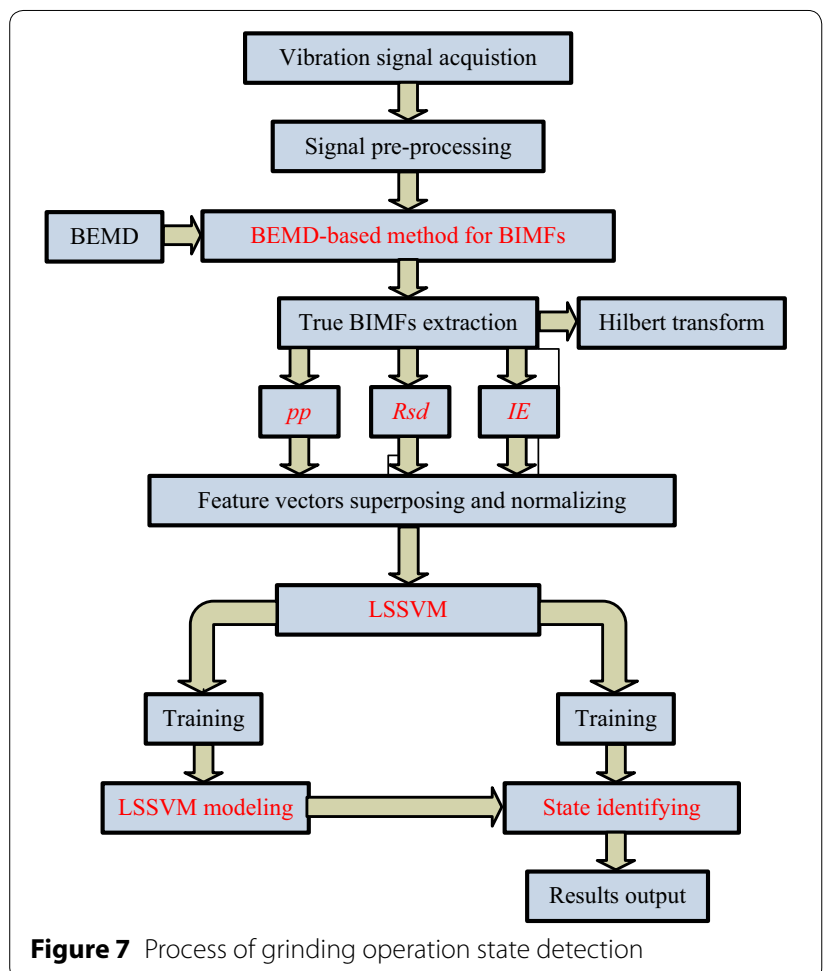

accurately revealed as corresponding with the same frequency components, i.e., $50 \mathrm{rad} / \mathrm{s}$ and $100 \mathrm{rad} / \mathrm{s}$, as set in the previous signal.

\subsection{Extraction of the Chatter Feature Vectors Based on BEMD}

Through the decomposition of BEMD and the extraction of true BIMFs, the chatter signal is decomposed into a series of true BIMFs and a residue. Another main objective of this paper is to extract appropriate feature vectors of each true BIMF and superpose them respectively to construct synthetic chatter feature vectors for chatter detection and identification. To compare and analyze the chatter feature vectors they should be normalized $[39,40]$ in order to keep the value between 0 and 1 . As described in Section 2.3, the peak to peak, real-time standard deviation and instantaneous energy are applied to the chatter signal and the superimposed and normalized feature vectors of the BIMFs, along with time, are shown in Figure 6. The $p p$ are plotted as blue solid lines, $R s d$ are plotted as a black dot lines, while $I E$ are plotted as a red-dashed lines.

In Figure 6, it is clearly seen that all feature vectors in various grinding states exhibit different behaviors and that the amplitude of the feature vectors is almost constant in a stable grinding state, while the amplitude drastically increases once the grinder turns into chatter. Moreover, the peak to peak and instantaneous energy fluctuation within a certain range when the grinder is in stable chatter state. The real-time standard deviation continuously increases with time and tends towards stability at the end, making it hard to exactly distinguish the transition state and chatter state. But all in all, it is feasible to clearly find out the onset of grinding chatter of great important to take reliable method to suppress the chatter. In summary, the peak to peak, real-time standard deviation and instantaneous energy are significantly distinct and could be used as a predictor for early grinding chatter detection.

\section{Grinding Experiments and Application of BEMD and LSSVM}

The ultimate purpose of this paper is to distinguish the onset of grinding chatter so that effective methods for chatter suppression can be applied as soon as possible. Therefore, a method based on BEMD and LSSVM is presented to detect and identify the grinding operation state. The process of this method is expressed in Figure 7.

\subsection{Grinding Experiments}

In order to further validate the feasibility of BEMD in grinding chatter detection, an experimental method was designed to acquire the various vibration states of different grinding parameters for the $\mathrm{CNC}$ guideway grinder KD4020X16 from Hangzhou Hangji Machine Tool Co., Ltd. The IEPE piezoelectric acceleration sensors with a supporting dynamic signal test and analysis system called 
TST5912 was used to collect the grinding vibration signal, as shown in Figure 8.

In practice, the grinder is more sensitive to the rotational speed, feeding speed and grinding depth of the grinding wheel, which contributes to the unbalance of the grinding vibration. The experiment was therefore carried out in following steps:

Firstly, keep the feeding speed of the workpiece and grinding depth of the wheel constant, and then gradually increase the rotational speed.

Secondly, keep the feeding speed and rotational speed constant, and then gradually increase the depth of grinding.

Lastly, keep the rotational speed and depth of grinding constant, and then gradually increase the feeding speed.

The resulting parameters are listed in Table 3.

According to the experimental conditions shown in Table 3, eight piezoelectric acceleration sensors are used to test the vibration acceleration signals of grinding wheel spindle, motor and machine column in various directions. The position of sensors and corresponding sensitivity are given in Table 4 .

Using the above steps, we collected and recorded corresponding signals until 80 groups of grinding signals were obtained, where 45 groups were in a stable grinding state, while 35 groups are in a chatter state. It has been proven that chatter of the wheel spindle is more significantly in the $X$-direction and $Z$-direction compared to the $Y$-direction based upon the practical experience and analysis of a considerable portion of the experimental data. For convenience of presentation, this paper selects parts of the $X$-direction and $Z$-direction chatter data to construct a complex-valued signal and then eliminates its noise based on the wavelet transform [41, 42]. The newly constructed complex-valued signal is shown in Figure 9, where the blue solid lines represent the $Z$-direction part and the red-dashed lines represent the $X$-direction.

From Figure 9, it is seen that the grinding chatter emerges at about 6-14 $\mathrm{s}$ and the transitional phase remains at almost $8 \mathrm{~s}$. It is clearly seen that the amplitude of the vibration signal rapidly expands when the grinder turns into chatter, then the amplitude becomes steady when the grinder gets into stable chatter. However, the signal vibrates more markedly compared with the stable grinding state.

\subsection{Application of BEMD to Experimental Chatter Signals} As previously discussed, the BEMD demonstrates its powerful capability in terms of processing the twodimensional simulation chatter signal and extracting true BIMFs from it. Moreover, the simulation results illustrate the phase information and synchronization between real

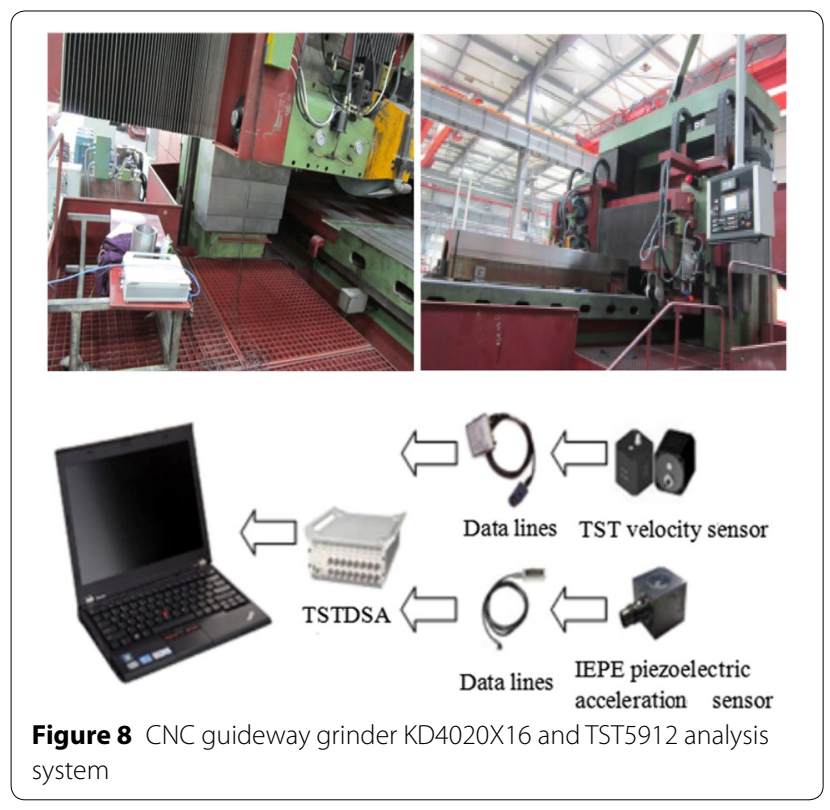

Table 3 Grinding parameters

\begin{tabular}{ll}
\hline Parameter & Value \\
\hline Grinding wheel material & Green silicon carbide \\
Size of wheel $(\mathrm{mm} \times \mathrm{mm})$ & $\phi 600 \times 150$ \\
Work-piece material & Gray cast iron 250 \\
Size of workpiece $(\mathrm{mm} \times \mathrm{mm} \times \mathrm{mm})$ & $3050 \times 500 \times 500$ \\
Rotational speed $(\mathrm{r} / \mathrm{min})$ & $700 \sim 1100$ \\
Feeding speed $(\mathrm{m} / \mathrm{s})$ & $0.381,0.254,0.210$ \\
Grinding depth $(\mu \mathrm{m})$ & $5,10,15$ \\
\hline
\end{tabular}

Table 4 Position of sensors and corresponding sensitivity

\begin{tabular}{lcl}
\hline Label of sensors & Sensitivity $\mathbf{( m V / g )}$ & Position \\
\hline 1 & 9.9 & Column Z \\
2 & 10.6 & Column $X$ \\
3 & 10.4 & Spindle $Z$ \\
4 & 10.1 & Spindle $Y$ \\
5 & 10.4 & Motor $Z$ \\
6 & 10.5 & Motor $X$ \\
7 & 10.2 & Motor $Y$ \\
8 & 10.1 & Column Y \\
\hline
\end{tabular}

and imaginary parts of the BIMFs, which is of significant value when applying the BEMD method to practical chatter. The BEMD is applicable to decompose the above experimental signal, which sets 64 projection directions at 10 iterations. Then the extraction criterion based on correlation coefficient could be applied to the extracted 


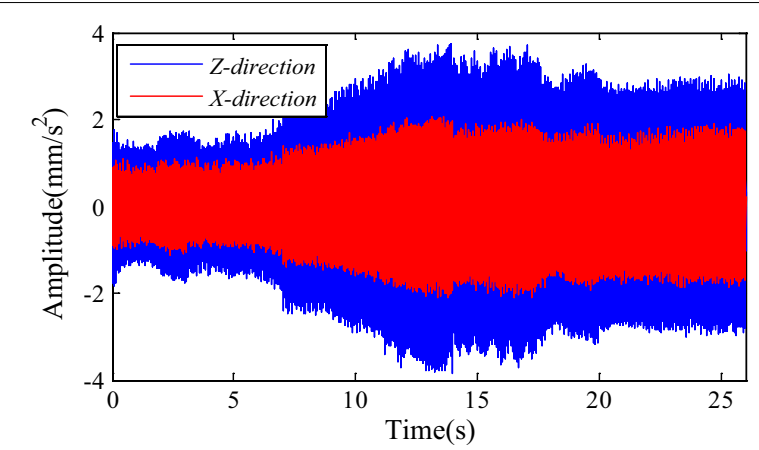

Figure 9 Newly constructed complex-valued signal from experimental data

Table 5 Correlation coefficients of the experimental BIMFs

\begin{tabular}{lll}
\hline No. & BIMFs & $\begin{array}{l}\text { Correlation } \\
\text { coefficient }\end{array}$ \\
\hline 1 & BIMF $_{1}$ & 0.9656 \\
2 & $\mathrm{BIMF}_{2}$ & 0.0700 \\
3 & $\mathrm{BIMF}_{3}$ & 0.0336 \\
4 & $\mathrm{BIMF}_{4}$ & 0.0156 \\
5 & $\mathrm{BIMF}_{5}$ & 0.0032 \\
6 & $\mathrm{BIMF}_{6}$ & 0.0016 \\
7 & $\mathrm{BIMF}_{7}$ & 0.0021 \\
8 & $\mathrm{BIMF}_{8}$ & 0.0014 \\
\hline
\end{tabular}

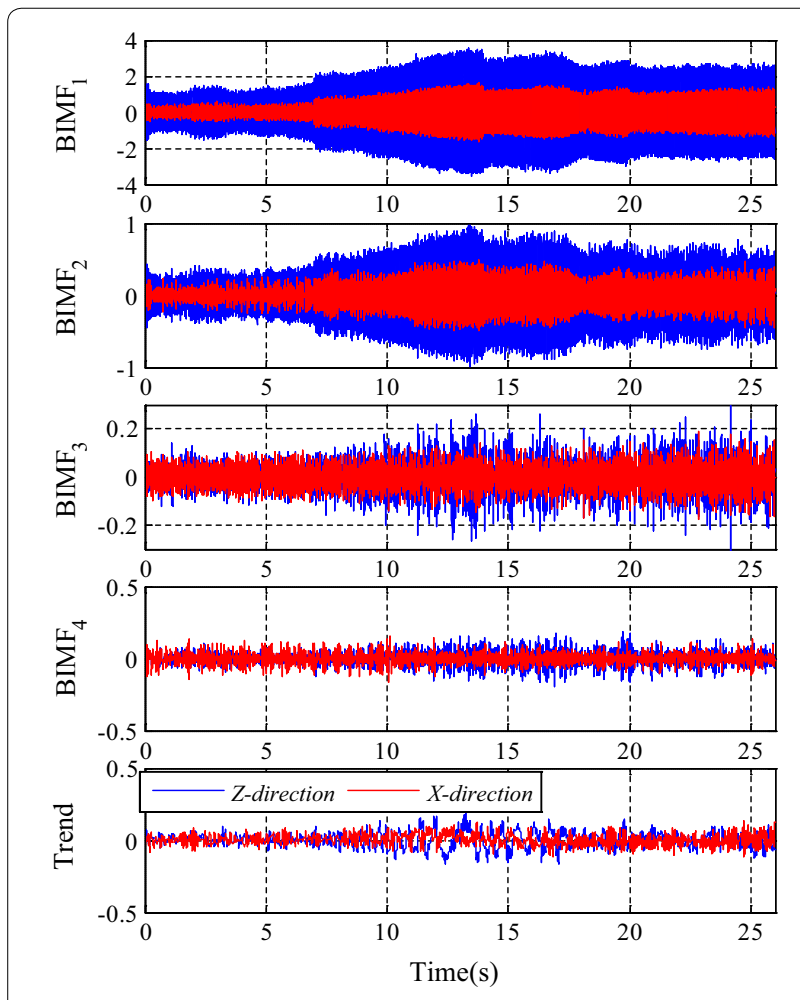

Figure 10 True BIMFs of the experimental chatter signal
BIMFs so that the correlation coefficients are shown in Table 5.

From Table 5, it is clearly seen that the first four BIMFs which have a relatively high correlation with the original signals should be reserved. The other four BIMFs have to be removed and classified as a part of the residual so the first three BIMFs could be recognized as the true BIMFs, which contain the main frequency components of the signal. The resulting decomposition is shown in Figure 10, where the blue solid lines represent the real part while the red-dashed lines represent the imaginary parts of the BIMFs.

According to Figure 10, it is clearly seen that phase shifting between the real parts and imaginary parts of the true BIMFs are well preserved and detected. Thus, the CCF of each true BIMF could be obtained and then the phase parameter estimated from the CCF, as shown in Table 6.

Then the amplitude and frequency components could be calculated by performing the Hilbert transform on each true BIMF to obtain the marginal spectrum, as shown in Figure 11.

In Figure 11, the marginal spectrum of both the $Z$-direction and $X$-direction shows the same frequency components, which represent about $300 \mathrm{~Hz}, 580 \mathrm{~Hz}$, $1200 \mathrm{~Hz}$ and $1400 \mathrm{~Hz}$. Yet the Z-direction BIMFs have a larger amplitude relative to the $X$-direction, and signal vibrates more significantly in the $Z$-direction according to the practical data.

\subsection{Chatter Feature Vectors Extraction for the Experimental Signal}

Based on the same principle and method, the feature vectors could also be extracted from the experimental true BIMFs, where the superimposed and normalized $p p$, Rsd and $I E$ of real and imaginary parts of the BIMFs, along with time, are shown in Figure 12. The $p p$ of the BIMFs are plotted as blue solid lines, $R s d$ are plotted as black dot lines, while $I E$ are plotted as red-dashed lines.

We can see that the behavior of these three feature vectors exhibit the same change in regulation as the previous simulation chatter signal in Section 3.3, that it can initially find out the onset time of chatter is nearly $6 \mathrm{~s}$. However, in order to detect and identify the onset of grinding chatter in time, it is very crucial to extract feature vectors during an early stage of its development. Therefore, the chatter feature vectors could be selected at the maximum value of the transition state. Then the feature vectors of the 80 collected grinding signals could be calculated and are shown in Figure 13, after superposing and normalizing, where the first 45 signals are stable grinding and the latter 35 signals are chatter grinding. The $p p$ is plotted as 
Table 6 Phase and maximum of CCF from each of the experimental BIMFs

\begin{tabular}{lcccc}
\hline Parameter & BIMF $_{\mathbf{1}}$ & $\mathbf{B I M F}_{\mathbf{2}}$ & $\mathbf{B I M F}_{\mathbf{3}}$ & $\mathbf{B I M F}_{\mathbf{4}}$ \\
\hline Estimated phase (rad) & 0.0562 & 0.0141 & 0.0350 & 0.0562 \\
Maximum of CCF & 6517.1 & 148.55 & 74.647 & 7.1760 \\
\hline
\end{tabular}

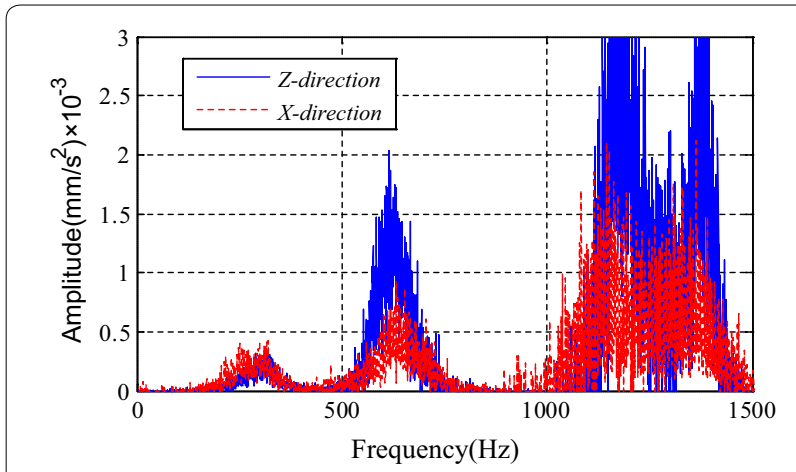

Figure 11 Marginal spectrum of the Z-direction signal and $X$-direction signal

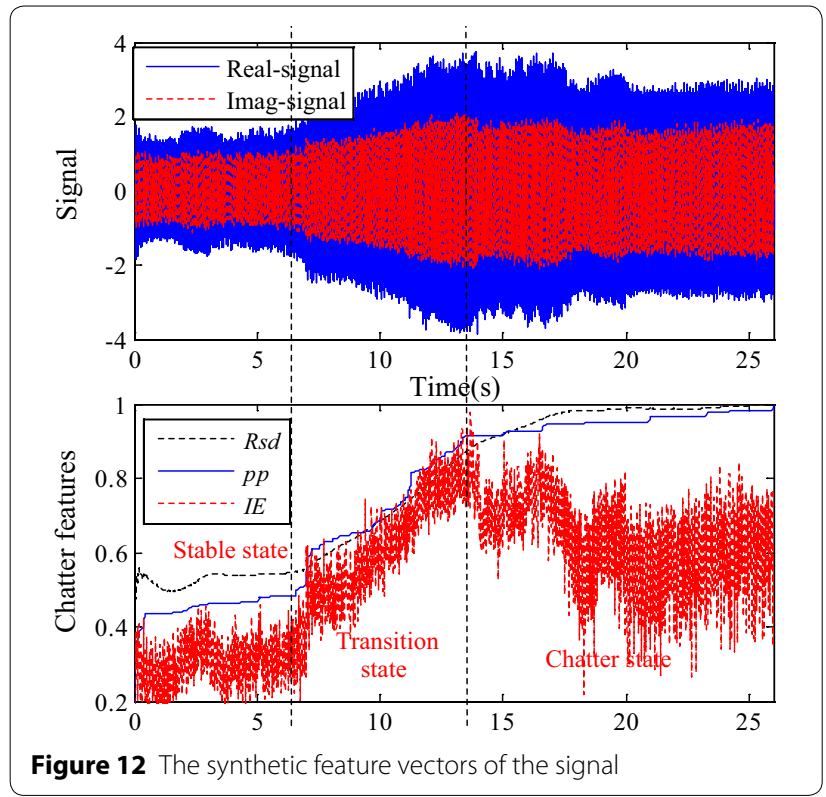

blue solid line, $R s d$ is plotted as black dot line, while $I E$ is plotted as red-dashed line.

From Figure 13, it is seen that the $p p, R s d$ and $I E$ of chatter grinding are increased to a different degree compared with stable grinding, that it is considered as the significant characteristic to distinguish the grinding state. Therefore, they are all employed as input data for the LSSVM.

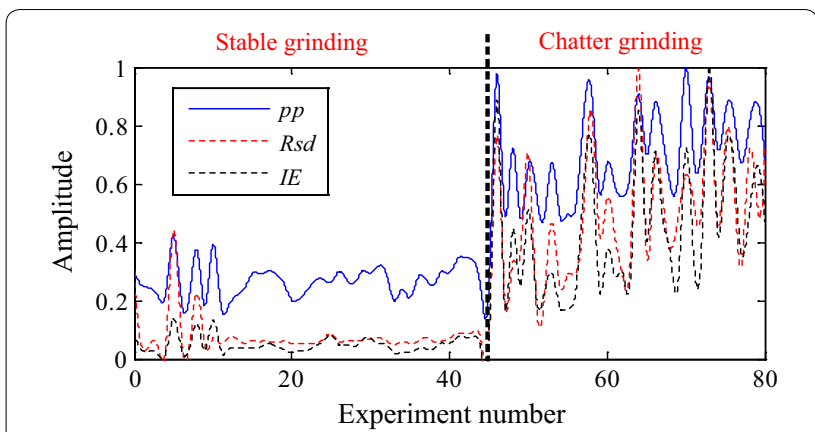

Figure 13 Features of 80 experimental signals

\subsection{LSSVM Model Prediction}

In accordance with the theory of LSSVM, the model should be established using training samples, which utilize feature vectors of 30 stable signals and 25 chatter signals at a random as the training set, with the remaining 15 stable signals and 10 chatter signals used for testing. The training of the LSSVM is performed in Matlab and the process of LSSVM training is actually the learning process from the expert knowledge mentioned in Section 2.2. Thus, two classifiers should be defined where -1 represents stable state and +1 represents chatter state. It is important to choose a suitable kernel function as it can strongly influence the reliability of the LSSVM results. In this paper, after a grid search and cross validation, an RBF kernel function was selected which sets the penalty coefficient $C=1$ and $\varepsilon=0.1$. The grinding parameters, feature vectors, and corresponding prediction results are shown in Table 7.

According to the data in Table 7, the 14 stable grinding signals and the other 10 chatter grinding signals are exactly identified by the LSSVM model, while the third stable signal is wrong for a chatter signal which is mostly caused by the concentrated load shock. The accuracy of this prediction model is $96 \%$, which could be considered an appropriate model to be applied for chatter detection and identification in the $\mathrm{CNC}$ guideway grinder KD4020X16. For convenience of presentation, the prediction model can be made into a diagram form, as shown in Figure 14, where the red asterisks and blue circles represent feature vectors of the stable state and chatter state, respectively.

It is seen that Figure 14 is the visual expression of the LSSVM prediction model. It is clearly divided into two parts: a stable state area and a chatter state area. Moreover, the feature vector distribution of chatter is more extensive than the stable state. Thus, in view of this grinding machine, the feature vectors could be extracted out from the real-time acquired signals by BEMD method, and then tested by this LSSVM model which has 
Table 7 Prediction results of the LSSVM model

\begin{tabular}{|c|c|c|c|c|c|c|c|c|c|}
\hline \multirow[t]{2}{*}{ Test No. } & \multicolumn{3}{|l|}{ Input } & \multirow[t]{2}{*}{ Target } & \multirow[t]{2}{*}{ Output } & \multirow[t]{2}{*}{ Result } & \multirow{2}{*}{$\begin{array}{l}\text { Rotational speed } \\
\text { ( } \mathrm{r} / \mathrm{min})\end{array}$} & \multirow{2}{*}{$\begin{array}{l}\text { Feeding speed } \\
(\mathrm{m} / \mathrm{s})\end{array}$} & \multirow{2}{*}{$\begin{array}{l}\text { Grinding } \\
\text { depth }(\mu \mathrm{m}\end{array}$} \\
\hline & $p p$ & $R s d$ & IE & & & & & & \\
\hline 1 & 01710 & 0.4626 & 1.9618 & 1 & 1 & Correct & 992 & 0.381 & 5 \\
\hline 2 & 0.0382 & 0.0111 & 0.0353 & -1 & -1 & Correct & 763 & 0.381 & 5 \\
\hline 3 & 0.0830 & 0.2870 & 0.2765 & -1 & 1 & Wrong & 1034 & 0.381 & 5 \\
\hline 4 & 0.0336 & 0.0113 & 0.0361 & -1 & -1 & Correct & 808 & 0.381 & 10 \\
\hline 5 & 0.0288 & 0.0115 & 0.0383 & -1 & -1 & Correct & 943 & 0.381 & 5 \\
\hline 6 & 0.4903 & 0.0631 & 0.3030 & 1 & 1 & Correct & 808 & 0.381 & 15 \\
\hline 7 & 0.0920 & 0.0163 & 0.0636 & -1 & -1 & Correct & 853 & 0.381 & 15 \\
\hline 8 & 0.0522 & 0.0176 & 0.0925 & -1 & -1 & Correct & 992 & 0.381 & 15 \\
\hline 9 & 0.1109 & 0.1937 & 0.5600 & 1 & 1 & Correct & 1074 & 0.254 & 15 \\
\hline 10 & 0.0327 & 0.0131 & 0.0363 & -1 & -1 & Correct & 804 & 0.254 & 10 \\
\hline 11 & 0.0851 & 0.1218 & 0.3987 & 1 & 1 & Correct & 803 & 0.254 & 15 \\
\hline 12 & 0.0394 & 0.0147 & 0.0513 & -1 & -1 & Correct & 844 & 0.254 & 15 \\
\hline 13 & 0.1162 & 0.2798 & 0.3863 & 1 & 1 & Correct & 936 & 0.254 & 10 \\
\hline 14 & 0.0421 & 0.0124 & 0.0570 & -1 & -1 & Correct & 989 & 0.254 & 5 \\
\hline 15 & 0.0470 & 0.0149 & 0.0996 & -1 & -1 & Correct & 1035 & 0.254 & 5 \\
\hline 16 & 0.1037 & 0.1263 & 0.3676 & 1 & 1 & Correct & 1040 & 0.254 & 15 \\
\hline 17 & 0.1602 & 0.5510 & 2.0361 & 1 & 1 & Correct & 760 & 0.210 & 5 \\
\hline 18 & 0.0325 & 0.0115 & 0.0245 & -1 & -1 & Correct & 760 & 0.210 & 10 \\
\hline 19 & 0.0431 & 0.0122 & 0.0427 & -1 & -1 & Correct & 853 & 0.210 & 5 \\
\hline 20 & 0.1031 & 0.1823 & 0.6225 & 1 & 1 & Correct & 893 & 0.210 & 10 \\
\hline 21 & 0.0471 & 0.0133 & 0.0702 & -1 & -1 & Correct & 935 & 0.210 & 5 \\
\hline 22 & 0.1787 & 0.3055 & 1.5177 & 1 & 1 & Correct & 936 & 0.210 & 10 \\
\hline 23 & 0.0507 & 00218 & 0.0884 & -1 & -1 & Correct & 1075 & 0.210 & 5 \\
\hline 24 & 0.1480 & 0.3681 & 0.9967 & 1 & 1 & Correct & 904 & 0.210 & 15 \\
\hline 25 & 0.0396 & 0.0139 & 0.0417 & -1 & -1 & Correct & 860 & 0.210 & 15 \\
\hline
\end{tabular}
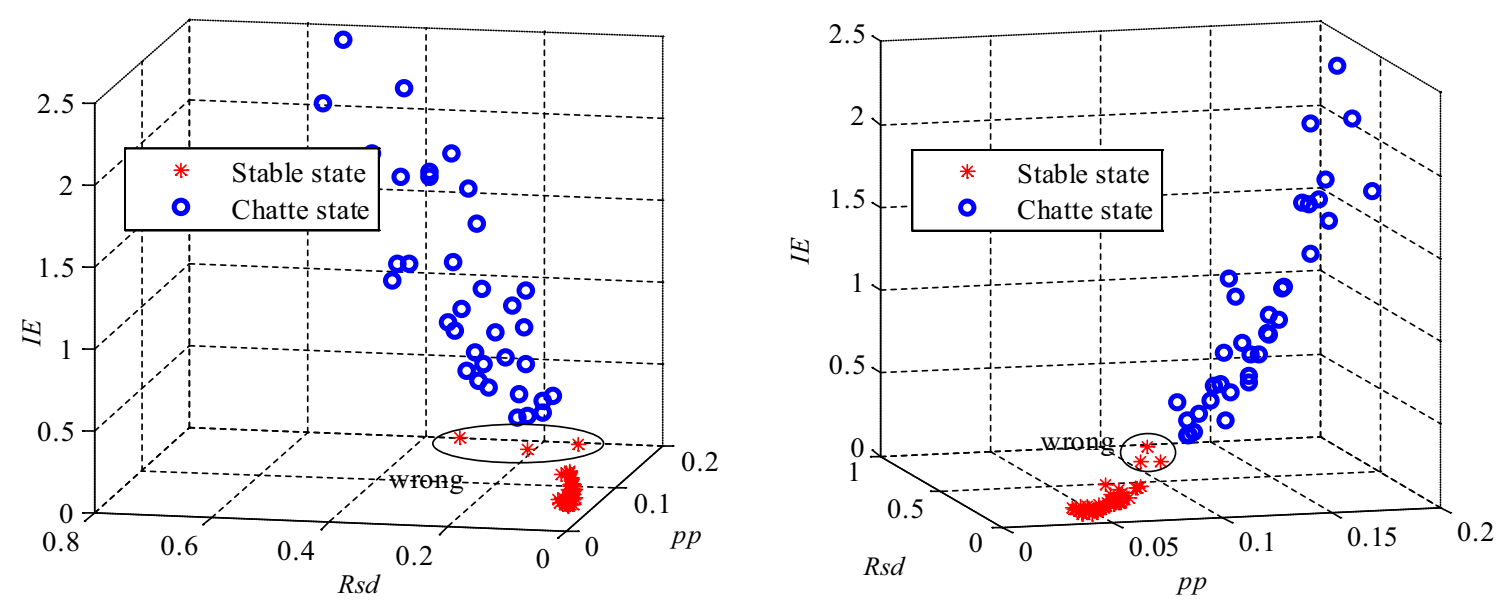

Figure 14 A diagram of feature vectors distribution

high accuracy and efficiency. If the feature vectors are in stable area, it means that the grinder is operating well. Otherwise, the grinding machine is suffering from the chatter, that effective measures should be taken immediately to reduce the damage of chatter. It is also necessary to collect more sample data as training set to improve 
identification accuracy of the LSSVM model. Using the diagram makes it more intuitive and convenient to judge which area the feature vector is in and then find out whether the grinder is in a stable grinding or chatter state. Consequently, the chatter detection and identification method based on BEMD and LSSVM in this paper has an excellent use for chatter prediction, which is robust under different grinding conditions.

\section{Conclusions}

In this paper, the BEMD was further investigated by processing a simulation chatter signal and an experimental chatter signal. Then the extraction criterion of true BIMFs based on the correlation coefficient successfully distinguished the true BIMFs from the spurious components. Phase shifting of the BIMFs were calculated, as well as the peak to peak, standard deviation, and instantaneous energy being presented as chatter feature vectors for detecting different vibration states of grinding. Lastly, the LSSVM model was established for grinding status classification based on feature vectors. From the research described above, the following conclusions can be drawn.

(1) The BEMD decomposes the simulation chatter signal derived from a grinding vibration signal generator and is validated by the experimental data which was collected from the CNC guideway grinder KD4020X16 in Hangzhou Hangji Machine Tool Co., Ltd. The results illustrate the suitability of BEMD in terms of processing non-stationary and nonlinear signals and indicating the phase shifting and synchronization information of signals. Meanwhile, the marginal spectrum accurately revealed the actual peculiarities of the signal.

(2) The extraction criterion of the true BIMFs based on the correlation coefficient is a reliable technique which successfully identifies and estimates the spurious components. It reserves the main frequency bands which are of great import to the extraction of the actual vibration mode and corresponding features of the time-frequency domain.

(3) The peak to peak, standard deviation, and kurtosis values are demonstrated as appropriate feature vectors for early grinding chatter detection.

(4) The prediction model based on BEMD and LSSVM shows its feasibility for chatter detection and identification, where the accuracy of this LSSVM model is $96 \%$.

For future work it should be notes that, although the feature vectors based on BEMD showed good performance, these vectors might not be the optimal choice. How to choose and estimate the feature vector is still a challenge for pattern recognition. Furthermore, the selection of the kernel function and the penalty coefficient is also a problem that needs further investigation. In addition, researching smart algorithms for optimization of the vector would be another interesting work.

\section{Authors' Contributions}

H-GC and C-SH was in charge of the whole trial; H-GC, J-YS and W-HC wrote the manuscript; Y-YY and J-CQ assisted with sampling and laboratory analyses. All authors read and approved the final manuscript.

\section{Author Details}

${ }^{1}$ Zhejiang Province's Key Laboratory of Reliability Technology for Mechanical and Electrical Product, Hangzhou 310018, China. ${ }^{2}$ Zhejiang Jiali Technology Co., Ltd., Hangzhou 311241, China. ${ }^{3}$ Hangzhou Hangji Machine Tool Co., Ltd., Hangzhou 310018, China.

\section{Authors' Information}

Huan-Guo Chen, born in 1977, is currently a doctor, Professor, Master's tutor at Zhejiang Province's Key Laboratory of Reliability Technology for Mechanical and Electrical Product, Zhejiang Sci-Tech University, China. She received her doctor degree from Northwestern Polytechnical University, China, in 2007. Her research interests include on line damage detection and fault diagnosis of intelligent structures.

Jian-Yang Shen, born in 1992, is currently an engineer at Zhejiang Jiali Technology Co., Ltd., China. He received his master degree on mechanical engineering from Zhejiang Sci-Tech University, China, in 2017.

Wen-Hua Chen, born in 1963, is currently a professor, Vice President and doctoral supervisor at Zhejiang Province's Key Laboratory of Reliability Technology for Mechanical and Electrical Product, Zhejiang Sci-Tech University, China. He received his doctor degree from Zhejiang University, China, in 1997. His research interests include on reliability and mechanism.

Chun-Shao Huang, born in 1976, is currently an engineer at Hangzhou Hangji Machine Tool Co., Ltd., China.

Yong-Yu Yi, born in 1991, is currently an engineer at Zhejiang Jiali Technology Co., Ltd., China. He received his master degree on mechanical engineering from Zhejiang Sci-Tech University, China, in 2017.

Jia-Cheng Qian, born in 1993, is currently a master candidate at Zhejiang Sci-Tech University, China.

\section{Competing Interests}

The authors declare that they have no competing interests.

\section{Funding}

Supported by National Natural Science Foundation of China (Grant No.

51475432), Zhejiang Provincial National Natural Science Foundation of China (Grant No. LZ13E050003), and State Key Program of National Natural Science of China (Grant Nos. U1234207, U1709210).

\section{Publisher's Note}

Springer Nature remains neutral with regard to jurisdictional claims in published maps and institutional affiliations.

Received: 16 January 2017 Accepted: 18 December 2018 Published online: 09 January 2019

\section{References}

[1] M Ahrens, R Fischer, M Dagen, et al. Abrasion monitoring and automatic chatter detection in cylindrical plunge grinding. Procedia CIRP, 2013(8): 374-378.

[2] L G Wang, X J Liu, Q F Jia. Studies and developments about grinding chatter of machine tools. Tianjin: Machine Tool \& Hydraulics, 2004.

[3] Wenfeng Ding, Barbara Linke, Yejun Zhu, et al. Review on monolayer CBN superabrasive wheels for grinding metallic materials. Chinese Journal of Aeronautics, 2017, 30(1): 109-134. 
[4] Wenzhong Li, Yujing Hu. Simulation analysis of ultrasonic vibration grinding of hard alloy. Journal of Qingdao University (Natural Science Edition), 2015, 28 (4): 66-71. (in Chinese)

[5] N H M Rozalli, N L Chin, Y A Yusof. Grinding characteristics of Asian originated peanuts (Arachishypogaea L.) and specific energy consumption during ultra-high speed grinding for natural peanut butter production. Journal of Food Engineering, 2015, 152(2): 1-7.

[6] X C Liu, F Chen, M S Fen, et al. Research of GCr15 bearing steel's surface roughness and grinding burn in ultra-high speed grinding. Modular Machine Tool \& Automatic Manufacturing Technique, 2016(9): 32-34. (in (hinese)

[7] H G Chen, JY Shen, W H Chen, et al. The bivariate empirical mode decomposition and its contribution to grinding chatter detection. Applied Sciences, 2017, 7(2): 145-163.

[8] Yao Liu, Xiufeng Wang, Jing Lin, et al. Early chatter detection in gear grinding process using servo feed motor current. The International Journal of Advanced Manufacturing Technology, 2016, 83(12): 1801-1810.

[9] Z L Yao, M Wang, T Zan, et al. Prediction method of grinding chatter based on ARIMA. Advanced Materials Research, 2014, 971-973 (9): 1288-1291.

[10] A Messaoud, CWeihs. Monitoring a deep hole drilling process by nonlinear time series modeling. Journal of Sound and Vibration, 2009, 321 (3-5): 620-630.

[11] E Kondo, H Ota, T Kawai. A new method to detect regenerative chatter using spectral analysis. Part 1. Basic study on criteria for detection of chatter. Journal of Manufacturing Science \& Engineering, 1997, 119(4A): 461-466.

[12] M C Yoon, D H Chin. Time series modeling and spectrum analysis for chatter mode in endmilling dynamics. The International Journal of Advanced Manufacturing Technology, 2006, 29(11): 1125-1133.

[13] I N Tansel, X Wang, P Chen, et al. Transformation in machining, Part 2. Evaluation of machining quality and Trans detection of chatter in turning by using s-transformation. International Journal of Machine Tools \& Manufacture, 2014, 46(a): 43-50.

[14] Zhehe Yao, Deqing Mei, Zichen Chen. On-line chatter detection and detection based on wavelet and support vector machine. Journal of Materials Processing Technology, 2010, 210(5): 713-719.

[15] J Gradisek, E Govekar, I Grabec. Using coarse-grained entropy rate to detect chatter in turning. Journal of Sound and Vibration, 1998, 214(5): 941-952.

[16] Gabriel Rilling, Partick Flandrin. Bivariate empirical mode decomposition. IEEE Signal Processing Letters, 2007, 14(12): 936-939.

[17] Wenxian Yang, Richard Court, Peter J Tavner. Bivariate empirical mode decomposition and its contribution to wind turbine condition monitoring. Journal of Sound and Vibration, 2011, 330(15): 3766-3782.

[18] Long Li, Jing Wei, Canbing Li. Prediction of load model based on artificial neural network. Transactions of China Electrotechnical Society, 2015, 30(8): 225-230. (in Chinese)

[19] Yuan Ren, Guangchen Bai. New neural network response surface methods for reliability analysis. Chinese Journal of Aeronautics, 2011, 24(1): 25-31.

[20] X Q Li, Y S Wong, A Y C Nee. A comprehensive identification of tool failure and chatter using a parallel multi-ART2 neural network. Journal of Manufacturing Science and Engineering, 1998, 120(2): 433-442.

[21] I Bediaga, J Muñoa, J Hernández, et al. An automatic spindle speed selection strategy to obtain stability in high-speed milling. International Journal of Machine Tools and Manufacture, 2009, 49(5): 384-394.

[22] YT Jiang, C L Zhang. Hybrid HMM/SVM method for predicting of cutting chatter. Proceedings of the SPIE-The International Society for Optical Engineering, 2006, 6280: 404-411.

[23] Qing Wang, Weiqi Qian, Kaifeng He. Unsteady aerodynamic modeling at high angles of attack using support vector machines. Chinese Journal of Aeronautics, 2015, 28(3): 659-668.

[24] Jianyang Shen. An online BEMD and LSSVM-based grinding chatter detection method for large grinding machine. Zhejiang: Zhejiang Sci-Tech University, 2017. (in Chinese)

[25] C W Hsu, C J Lin. A comparison of methods formulticlass support vector support vector machines. IEEE Transactions on Neural Networks, 2002, 13(2): 415-425.
[26] N E Huang, Z Shen, S R Long, et al. The empirical mode decomposition and the Hilbert spectrum for nonlinear and non-stationary time series analysis. Proceedings Mathematical Physical \& Engineering Sciences, 1998, 454(1971): 903-995.

[27] Changfu Liu, Lida Zhu, Chenbing Ni. The chatter identification in end milling based on combining EMD and WPD. The International Journal of Advanced Manufacturing Technology, 2017, 91 (9-12): 3339-3348.

[28] H G Chen, Y J Yan, W H Chen, et al. Early damage detection in composite wingbox structures using Hilbert-Huang Transform and Genetic Algorithm. International Journal of Structural Health Monitoring, 2007, 6(4): 281-297.

[29] Z H Zhu, Y L Sun, J I Yu. Short-term load forecasting research based on EMD and SVM. High Voltage Engineering, 2007, 33(5): 118-122.

[30] S J Rong, L Pan, XX Huang, et al. The influence of training step on price forecasting based on least squares support vector machine. Applied Mechanics \& Materials, 2014, 530-531: 621-624.

[31] Xin Ma. Power transformer fault diagnosis based on least squares support vector machine and particle swarm optimization. Applied Mechanics \& Materials, 2011, 50-51: 624-628.

[32] Q Wu. Monthly run off forecasting research based on wavelet transform and LSSVM. Xinjiang: Xinjiang University, 2015. (in Chinese)

[33] Zhang Fei, Xinfeng Ge, Luoping Pan, et al. Shaft run-outs' peak to peak value calculation method for a hydraulic power unit under stable conditions. Journal of Vibration and Shock, 2015, 34(21): 170-174.

[34] Bai Yu, Huang Zhigang, Li Rui. Analyze of algorithm based on estimating navigation satellite measurement noise. Annual Conference on Ship Communication and Navigation, 2008, 12(4): 11-16. (in Chinese)

[35] Yafu Yao, Zhang Xing. Fault diagnosis approach for roller bearing based on EMD momentary energy entropy and SVM. Journal of Electronic Measurement and Instrumentation, 2013, 27(10): 957-962.

[36] Xuelong Li, Zhonghui Li, Enyuan Wang, et al. Analysis of natural mineral earthquake and blast based on Hilbert-Huang transform (HHT). Journal of Applied Geophysics, 2016, 128: 79-86.

[37] Wang Ming, Fen Meng, Yao Ziliang, et al. Prediction of grinding chatter based on the ARIMA. Journal of Beijing University of Technology, 2016, 42: 609-613.

[38] Yingxia Luo, Ma Jun, Qingsong Zhu. A method for phase difference measurement with correlation function based on Matlab. Sci/Tech Information Development \& Economy, 2003, 13(7): 1-2.

[39] Hanguang Han, Congzhong Cai. Comparison study of normalization of feature vector. Engineer and Application, 2009, 45(22): 117-119.

[40] Rui LIN. An improved fast algorithm for the fractional Fourier transform based on the method of the dimensional normalization. Journal of Jiangxi Normal University (Natural Sciences Edition), 2016, 40 (01): 71-76. (in Chinese)

[41] Wang Nan, Jinsong Du. Application of wavelet de-noising in unsteady vibration signal processing. Chinese Journal of Scientific Instrument, 2001.

[42] J H CAl, J Li. Suppression of power line interference on MT signals based on the frequency domain wavelet method. Geology and Exploration, 2015, 51(02): 353-359.

\section{Submit your manuscript to a SpringerOpen ${ }^{\circ}$ journal and benefit from:}

- Convenient online submission

- Rigorous peer review

- Open access: articles freely available online

- High visibility within the field

- Retaining the copyright to your article

Submit your next manuscript at $\boldsymbol{\nabla}$ springeropen.com 